\title{
Guaranteed Passive Parameterized Macromodeling by Using Sylvester State-Space Realizations
}

\author{
Elizabeth Rita Samuel, Luc Knockaert, Senior Member, IEEE, Francesco Ferranti, Member, IEEE, \\ and Tom Dhaene, Senior Member, IEEE
}

\begin{abstract}
A novel state-space realization for parameterized macromodeling is proposed in this paper. A judicious choice of the state-space realization is required in order to account for the assumed smoothness of the state-space matrices with respect to the design parameters. This technique is used in combination with suitable interpolation schemes to interpolate a set of statespace matrices, and hence the poles and residues indirectly, in order to build accurate parameterized macromodels. The key points of the novel state-space realizations are the choice of a proper pivot matrix and a well-conditioned solution of a Sylvester equation. Stability and passivity are guaranteed by construction over the design space of interest. Pertinent numerical examples validate the proposed Sylvester realization for parameterized macromodeling.
\end{abstract}

Index Terms-Sylvester equation, parameterized macromodeling, state-space matrices, rational approximation, interpolation.

\section{INTRODUCTION}

D URING a typical design process of electromagnetic (EM) systems, design space exploration, design optimization, and sensitivity analysis are usually performed, and this requires multiple simulations for different design parameter values (e.g., layout features). Parameterized macromodels are valuable tools to efficiently and accurately perform these design activities, while avoiding new measurements or simulations for each new parameter configuration. Parameterized macromodels are multivariate models that describe the complex behavior of EM systems, typically characterized by frequency (or time) and several geometrical and physical design parameters, such as layout or substrate features. Recently, parameterized macromodeling techniques able to guarantee overall stability and passivity have been proposed in [1][4]. The techniques described in [1] and [2] are based on the interpolation of a set of univariate macromodels, called root macromodels. This interpolation process of input-output systems leads to parameterization of the residues, but unfortunately not of the poles. Passive interpolation of the state-space matrices of a set of root macromodels is proposed in [3] and [4], providing an increased modeling capability with respect to [1] and [2], due to the parameterization of both poles and residues. Unfortunately, these methods are sensitive to issues related to the interpolation of state-space matrices [5], such

Manuscript received September 17. This work was supported by the Research Foundation Flanders (FWO).

Elizabeth Rita Samuel, Francesco Ferranti, Luc Knockaert and Tom Dhaene are with Ghent University - IBBT, Gaston Crommenlaan 8 Bus 201, B-9050 Gent, Belgium,email: \{elizabeth.ritasamuel, francesco.ferranti, luc.knockaert, tom.dhaene\}@ugent.be as the smoothness of the state-space matrices as a function of the parameters.

In this paper, we propose a novel state-space realization that is suitable to build accurate parameterized macromodels. The direct parameterization of poles and residues is avoided, due to their potentially non-smooth behavior with respect to the design parameters. The Vector Fitting (VF) technique is initially used to build a set of root macromodel for different combinations of design variables. Stability for each root macromodel is enforced by pole flipping [6], while passivity is checked and enforced by means of standard techniques (e.g., [6]-[9]). A conversion from a pole-residue form obtained by means of VF to a Sylvester realization is computed for each root macromodel. The key points of the Sylvester realization is the choice of a pivot or reference matrix and the obtention of a well-conditioned solution to the Sylvester equation. Since the same pivot matrix is used for all state-space realizations of the root macromodels, smooth variations of the state-space matrices with respect to the design parameters can be expected. The state-space matrices obtained from the Sylvester realization are used to obtain matrix solutions of the linear matrix inequalities (LMIs) pertaining to the positive-real or boundedreal lemma [10], and this information is then used to perform a passivity preserving interpolation of the state-space matrices. The computations can be carried out using the solution of LMIs or algebraic Riccati equations (AREs) to generate a descriptor state-space format that preserves positive-realness or bounded-realness. Finally, suitable interpolation schemes are used to build accurate parameterized macromodels which preserve stability and passivity.

Pertinent numerical examples validate the proposed Sylvester realization for macromodeling based on interpolation of state-space matrices.

\section{Parameterized Macromodeling}

Starting from a set of data samples $\left\{(s, \vec{p})_{k}, H(s, \vec{p})_{k}\right\}$, a set of frequency-dependent rational models is built for an initial selection of design space points by means of system identification techniques [11], in our case the VF technique [6]. The result of this initial procedure is a set of rational univariate macromodels, called root macromodels. Each root macromodel is related to a design space point $\vec{p}_{k}=\left(p_{k_{1}}^{(1)}, \ldots, p_{k_{M}}^{(M)}\right)$. Two data grids are used in the modeling process: an estimation grid and a validation grid. The estimation grid is utilized to build the root macromodels that, combined with suitable interpolation schemes, are able to provide stable and passive 
parameterized macromodels. The validation grid is utilized to assess the interpolation capability of the parameterized macromodel and its capability of describing the system under study in points of the design space previously unused for the construction of the root macromodels.

Suppose we have a set of root macromodels $\mathscr{S}_{\vec{p}_{k}}, \quad k=$ $1, \ldots, N$ with given minimal realizations

$$
\mathscr{S}_{\vec{p}_{k}} \equiv\left[\begin{array}{cc}
A_{\vec{p}_{k}} & B_{\vec{p}_{k}} \\
C_{\vec{p}_{k}} & D_{\vec{p}_{k}}
\end{array}\right]
$$

state-space equations

$$
\begin{aligned}
\dot{x} & =A_{\vec{p}_{k}} x+B_{\vec{p}_{k}} u \\
y & =C_{\vec{p}_{k}} x+D_{\vec{p}_{k}} u
\end{aligned}
$$

and transfer functions

$$
H_{\vec{p}_{k}}(s)=C_{\vec{p}_{k}}\left(s I-A_{\vec{p}_{k}}\right)^{-1} B_{\vec{p}_{k}}+D_{\vec{p}_{k}} .
$$

We assume in this paper that all realizations $\mathscr{S}_{\vec{p}_{k}}$ have the same McMillan degree $n$ [12] and number of ports $m \leq n$. We further suppose that all matrices $A_{\vec{p}_{k}}$ are Hurwitz stable i,e; every eigenvalue of $A_{\vec{p}_{k}}$ has strictly negative real part [13]. We propose a generic parameterized realization of the form

$$
\mathscr{S}(\vec{p}) \equiv\left[\begin{array}{cc}
A(\vec{p}) & B(\vec{p}) \\
C(\vec{p}) & D(\vec{p})
\end{array}\right]
$$

with $\vec{p}=\left(\vec{p}^{(1)}, \ldots, \vec{p}^{(M)}\right)$. The models $\mathscr{S}_{\vec{p}_{k}}$ can be considered as snapshots of $\mathscr{S}(\vec{p}) . \mathscr{S}_{\vec{p}_{k}}$ should be able to accurately model the system behavior as a function of the complex frequency $s$ and the vectorial parameter $\vec{p}$ while guaranteeing stability and passivity over the design space of interest.

\section{State-Space Realization For Parameterized MACROMODELING}

To obtain accurate parameterized macromodels by interpolation of the state-space matrices, the choice of the state-space realization is fundamental.

In this section, we will discuss the well-known Gilbert realization, balanced realization and barycentric realization, and then the proposed novel Sylvester realization, preceded by an important subsection on passive parameterized interpolation.

\section{A. Gilbert Realization}

The minimal state-space realization problem for linear time invariant (LTI) systems was first stated by Gilbert [14], who gave an algorithm for transforming a transfer function into a system of differential equations. The approach , of Gilbert is based on partial-fraction expansions.

$$
H(s)=R_{0, \vec{p}_{k}}+\sum_{n=1}^{N} \frac{R_{n, \vec{p}_{k}}}{s-z_{n, \vec{p}_{k}}}
$$

where $R_{n, \vec{p}_{k}}$ and $s-z_{n, \vec{p}_{k}}$ are respectively the model residues and poles, with $R_{0, \vec{p}_{k}}$ being the direct coupling constant. The poles and the residues are stamped directly in the $A(\vec{p})$ and $C(\vec{p})$ matrices using the Gilbert realization [14]. It is wellknown that model poles and residues are very sensitive to even small variations of the design parameters, resulting in quite irregular variations of each pole in the design space, e.g. bifurcation effects [15]. Since poles and residues may present a highly non-smooth behavior with respect to the design parameters, achieving a reasonable accuracy in parameterized macromodels built by interpolation of state-space matrices becomes difficult, due to the fact that pole and residue trajectories as a function of $\vec{p}$ are not well defined.

\section{B. Balanced Realization}

A minimal and stable realization is called balanced [16], [17], if the controllability and observability Gramians [16] are equal and diagonal. Every minimal system can be brought into balanced form. The balanced realization can be implemented using the Matlab function balreal. This routine uses the eigendecomposition of the product of the observability and controllability Gramians to construct the balancing transformation matrix.

The most interesting properties of balanced realizations is associated with the uniqueness properties of the balancing transformation [18]. As the eigenvalues (real and nonnegative) of the product of the controllability and observability Gramians, are distinct, then the balancing transformation matrix is unique. If, on the other hand, two or more eigenvalues are repeated, then their corresponding eigenvectors can be rotated arbitrarily in the corresponding eigenspace. Thus as stated in [5], [18], [19], uniqueness is guaranteed up to a sign and it may affect the smoothness of the state-space matrices as functions of the design parameters.

\section{Barycentric Realization}

In what follows, the barycentric realization [15], [20] is described. The transfer function of the macromodel related to a generic point $\vec{p}=\left(p_{k_{1}}^{(1)}, \ldots, p_{k_{M}}^{(M)}\right)$ in the design space is converted from the rational pole residue form

$$
H(s)=R_{0, \vec{p}_{k}}+\sum_{n=1}^{N} \frac{R_{n, \vec{p}_{k}}}{s-z_{n, \vec{p}_{k}}}
$$

obtained by means of VF, into the barycentric realization [15], [21]

$$
H(s)=\frac{F_{0, \vec{p}_{k}}+\sum_{n=1}^{N} F_{n, \vec{p}_{k}} \phi_{n}(s)}{f_{0, \vec{p}_{k}}+\sum_{n=1}^{N} f_{n, \vec{p}_{k}} \phi_{n}(s)}
$$

with basis function

$$
\phi_{n}(s)=\frac{1}{s-a_{n}}, n=1, \ldots, N
$$

and where the barycentric basis poles $\left\{a_{n}\right\}_{n=1}^{N}$ are fixed and do not depend on $\vec{p}_{k}$. The barycentric realization (8) can be split into a numerator and a denominator, i.e;

$$
H(s)=\frac{N_{\vec{p}_{k}}(s)}{\Delta_{\vec{p}_{k}}(s)}
$$

where

$$
\begin{aligned}
& N_{\vec{p}_{k}}(s)=F_{0, \vec{p}_{k}}+\sum_{n=1}^{N} F_{n, \vec{p}_{k}} \phi_{n}(s) \\
& \Delta_{\vec{p}_{k}}(s)=f_{0, \vec{p}_{k}}+\sum_{n=1}^{N} f_{n, \vec{p}_{k}} \phi_{n}(s)
\end{aligned}
$$


This factorization can be seen as a special case of the so called right coprime factorization [22]. A state-space realization for each root macromodel is obtained by means of (11)-(12). First, we construct two separate state-space realizations for the numerator and denominator. We have

$$
\begin{aligned}
N_{\vec{p}_{k}}(s) & =\left[\begin{array}{ll}
\tilde{A}_{\vec{p}_{k}}^{\text {num }} & \tilde{B}_{\vec{p}_{k}}^{\text {num }} \\
\tilde{C}_{\vec{p}_{k}}^{\text {num }} & \tilde{D}_{\vec{p}_{k}}^{\text {num }}
\end{array}\right] \\
\Delta_{\vec{p}_{k}}(s) & =\left[\begin{array}{ll}
\tilde{A}_{\vec{p}_{k}}^{\text {den }} & \tilde{B}_{\vec{p}_{k}}^{\text {den }} \\
\tilde{C}_{\vec{p}_{k}}^{d e n} & \tilde{D}_{\vec{p}_{k}}^{d e n}
\end{array}\right]
\end{aligned}
$$

where

- $A_{\vec{p}_{k}}^{n u m}=A_{\vec{p}_{k}}^{\text {den }}=\operatorname{blkdiag}\left\{a_{n} I_{P}\right\}, n=1, \ldots, N$, where $I_{P}$ is the identity matrix of order $P$

- $B_{\vec{p}_{k}}^{\text {num }}=B_{\vec{p}_{k}}^{\text {den }}=\left[I_{P}, \ldots, I_{P}\right]^{T}$ is a block-column matrix obtained by stacking $N$ identity matrices $I_{P}$

- $C_{\vec{p}_{k}}^{n u m}=\left[F_{1, \vec{p}_{k}}, \ldots, F_{N, \vec{p}_{k}}\right]$

- $C_{\vec{p}_{k}}^{d e n}=\left[f_{1, \vec{p}_{k}} I_{P}, \ldots, f_{N, \vec{p}_{k}} I_{P}\right]$

- $D_{\vec{p}_{k}}^{n u m}=F_{0, \vec{p}_{k}}$

- $D_{\vec{p}_{k}}^{d e n}=f_{0, \vec{p}_{k}} I_{P}$

Remark that, in case some of the basis poles in the set of $\left\{a_{n}\right\}_{n=1}^{N}$ are complex, the above state-space matrices are complex. However, standard state-space coordinate transformations can be applied such that the resulting realization is real. The matrix $A_{\vec{p}_{k}}^{n u m}=A_{\vec{p}_{k}}^{d e n}$ can be seen as a pivot matrix for the barycentric realization. Although the denominator function (12) is scalar, its realization has been chosen to have $P$ ports, in order to be compatible in size with the realization of (11). We have

$$
H(s)=N_{\vec{p}_{k}}(s) \Delta_{\vec{p}_{k}}(s)^{-1} I_{P}
$$

Then after some standard manipulations [15], we can write the final state-space realization of (10) as

$$
H(s)=\left[\begin{array}{cc}
\tilde{A}_{\vec{p}_{k}} & \tilde{B}_{\vec{p}_{k}} \\
\tilde{C}_{\vec{p}_{k}} & \tilde{D}_{\vec{p}_{k}}
\end{array}\right]
$$

with

$$
\begin{aligned}
\tilde{A}_{\vec{p}_{k}} & =A_{\vec{p}_{k}}^{d e n}-B_{\vec{p}_{k}}^{d e n}\left(D_{\vec{p}_{k}}^{d e n}\right)^{-1} C_{\vec{p}_{k}}^{d e n} \\
\tilde{B}_{\vec{p}_{k}} & =B_{\vec{p}_{k}}^{d e n}\left(D_{\vec{p}_{k}}^{d e n}\right)^{-1} \\
\tilde{C}_{\vec{p}_{k}} & =C_{\vec{p}_{k}}^{n u m}-D_{\vec{p}_{k}}^{n u m}\left(D_{\vec{p}_{k}}^{d e n}\right)^{-1} C_{\vec{p}_{k}}^{d e n} \\
\tilde{D}_{\vec{p}_{k}} & =D_{\vec{p}_{k}}^{n u m}\left(D_{\vec{p}_{k}}^{d e n}\right)^{-1}
\end{aligned}
$$

The barycentric realization (17) for each root macromodel can be computed using different methods. The robustness of the conversion is an important aspect. The computation of the barycentric realization (17) requires the identification of the matrices $C_{\vec{p}_{k}}^{n u m}$ and $C_{\vec{p}_{k}}^{d e n}$, which can be found using any of the approaches described below.

\section{1) Pole placement approach:}

An interesting and robust approach to compute the barycentric realization uses a pole placement technique [23]. The matrix

$$
\tilde{A}_{\vec{p}_{k}}=A_{\vec{p}_{k}}^{d e n}-B_{\vec{p}_{k}}^{d e n}\left(D_{\vec{p}_{k}}^{d e n}\right)^{-1} C_{\vec{p}_{k}}^{d e n}
$$

requires the identification of $C_{\vec{p}_{k}}^{d e n}$ which can be computed using the technique proposed in [23]. Another interesting approach based on the solution of a Sylvester equation can be found in [24]. Some assumptions concerning observability and controllability must be satisfied for these approaches and for the uniqueness of the solution [23], [24]. The matrix $C_{\vec{p}_{k}}^{\text {den }}$ is used to place the eigenvalues of the $\tilde{A}_{\vec{p}_{k}}$ matrix, and therefore the poles of the root macromodels in such a way that they are equal to $z_{n, \vec{p}_{k}}$. Once $C_{\vec{p}_{k}}^{d e n}$ is obtained, the next step is to compute the $C_{\vec{p}_{k}}^{n u m}$ matrix. Each block $F_{n, \vec{p}_{k}}, n=1, \ldots, N$ can be easily computed by means of [15]

$$
F_{n, \vec{p}_{k}}=f_{n, \vec{p}_{k}} H\left(a_{n}\right) .
$$

\section{2) Linear system approach:}

The matrix $C_{\vec{p}_{k}}^{\text {den }}$ is computed solving a linear system in [15]. This approach uses the solution of a linear system that contains the products of poles and becomes ill-conditioned if the order of the system is high and the bandwidth of interest is large. Once $C_{\vec{p}_{k}}^{d e n}$ is obtained, the computation of $C_{\vec{p}_{k}}^{\text {num }}$ is done as in the pole placement approach described above.

1) Passive Parameterized Interpolation: When the macromodel is utilized in a circuit simulator for transient analysis, stability and passivity are the fundamental properties to be guaranteed. It is known that while a passive system is also stable, the reverse is not necessarily true. A passive system denotes a system that is incapable of generating energy, and hence one that can only absorb energy from the sources used to excite it [25]. Passivity is an important property to satisfy because stable, but not passive macromodels can produce unstable systems when connected to other stable, even passive, loads.

The interpolation of state-space matrices does not result in the preservation of stability and passivity over the design space even if the root macromodels (1) are stable and passive. First , we need to define what we mean by passive interpolation. Since each root macromodel $\mathscr{S}_{\vec{p}_{k}}$ is passive, the bounded real lemma or positive real lemma [25] states that this is the case if there exists a positive definite symmetric matrix $P_{p_{k}}$ such that the (LMI) [20], [26], [27]

$$
\mathscr{L}_{\vec{p}_{k}}=\left[\begin{array}{ccc}
A_{\vec{p}_{k}}^{T} P_{p_{k}}+P_{p_{k}} A_{\vec{p}_{k}} & P_{\vec{p}_{k}} B_{\vec{p}_{k}} & C_{\vec{p}_{k}}^{T} \\
\left(P_{p_{k}} B_{\vec{p}_{k}}\right)^{T} & -I & D_{\vec{p}_{k}}^{T} \\
C_{\vec{p}_{k}} & D_{\vec{p}_{k}} & -I
\end{array}\right] \leq 0
$$

for S-parameters [28] or

$$
\mathscr{L}_{\vec{p}_{k}}=\left[\begin{array}{cc}
A_{\vec{p}_{k}}^{T} P_{\vec{p}_{k}}+P_{\vec{p}_{k}} A_{\vec{p}_{k}} & P_{\vec{p}_{k}} B_{\vec{p}_{k}}-C_{\vec{p}_{k}}^{T} \\
\left(P_{\vec{p}_{k}} B_{\vec{p}_{k}}-C_{\vec{p}_{k}}^{T}\right)^{T} & -D_{\vec{p}_{k}}-D_{\vec{p}_{k}}^{T}
\end{array}\right] \leq 0
$$

for Y-parameters is satisfied.

Solving the LMI can be replaced by equivalently solving an ARE, which is known to be a more efficient approach [29], [30] as the number of operations required to solve a Riccati equation is $O\left(n^{3}\right)$, while the cost of solving an equivalent LMI is $O\left(n^{6}\right)$. Thus for high orders it is advisable to solve using ARE as it is computationally cheaper in comparison with LMI. 
For the S-parameters and Y-parameters, the AREs can respectively be written as

$$
\begin{gathered}
A_{\vec{p}_{k}}^{T} P_{\vec{p}_{k}}+P_{\vec{p}_{k}} A_{\vec{p}_{k}}+C_{\vec{p}_{k}}^{T} C_{\vec{p}_{k}}+ \\
\left(P_{\vec{p}_{k}} B_{\vec{p}_{k}}+C_{\vec{p}_{k}}^{T} D_{\vec{p}_{k}}\right) W_{s}\left(P_{\vec{p}_{k}} B_{\vec{p}_{k}}+C_{\vec{p}_{k}}^{T} D_{\vec{p}_{k}}\right)^{T}=0
\end{gathered}
$$

and

$A_{\vec{p}_{k}}^{T} P_{\vec{p}_{k}}+P_{\vec{p}_{k}} A_{\vec{p}_{k}}+\left(P_{\vec{p}_{k}} B_{\vec{p}_{k}}-C_{\vec{p}_{k}}^{T}\right) W_{a}\left(P_{\vec{p}_{k}} B_{\vec{p}_{k}}-C_{\vec{p}_{k}}^{T}\right)^{T}=0$

if $W_{s}=\left(I_{m}-D_{\vec{p}_{k}}^{T} D_{\vec{p}_{k}}\right)^{-1}$ and $W_{a}=\left(D_{\vec{p}_{k}}+D_{\vec{p}_{k}}^{T}\right)^{-1}$ exist, i.e; $\left(I_{m}-D_{\vec{p}_{k}}^{T} D_{\vec{p}_{k}}\right)>0$ and $D_{\vec{p}_{k}}+D_{\vec{p}_{k}}^{T}>0$.

Once the matrix $P_{\vec{p}_{k}}$ is obtained for each root macromodel, it is possible to convert the transfer function from the form (4) to the descriptor state-space form

$$
H_{\vec{p}_{k}}(s)=C_{\vec{p}_{k}}\left(s P_{\vec{p}_{k}}-P_{\vec{p}_{k}} A_{\vec{p}_{k}}\right)^{-1} P_{\vec{p}_{k}} B_{\vec{p}_{k}}+D_{\vec{p}_{k}} .
$$

which will be useful for obtaining the passive parameterized interpolation.

Next, consider a positive interpolation kernel [31] $\mu_{p_{k}}(\vec{p})$ satisfying [32]

$$
\begin{gathered}
\mu_{p_{k}}(\vec{p}) \geq 0, \quad \mu_{p_{k}}\left(\vec{p}_{l}\right)=\delta_{p_{k}, l} \\
\sum_{p_{k}} \mu_{p_{k}}(\vec{p})=1
\end{gathered}
$$

We assume that the interpolation kernel only depends on the design space grid points and not on the values of the functions to be interpolated. The kernel computation does not require the solution of a linear system to impose the interpolation constraints. In what follows, each interpolation kernel $\mu_{p_{k}}(\vec{p})$, is selected as in piecewise linear interpolation.

It is straightforward to prove that, if each state-space matrix is interpolated by a positive interpolation scheme with an interpolation kernel that depends locally on the data points, this is equivalent with the interpolation of the LMI (20) with the same interpolation scheme i.e;

$$
\mathscr{L}(\vec{p})=\sum_{p_{k}=1}^{N} \mu_{p_{k}}(\vec{p}) \mathscr{L}_{p_{k}}
$$

Hence if we parameterize all entries of the $P_{\vec{p}_{k}} A_{\vec{p}_{k}}, P_{\vec{p}_{k}} B_{\vec{p}_{k}}, C_{\vec{p}_{k}}, D_{\vec{p}_{k}}, P_{\vec{p}_{k}}$ matrices as

$$
\begin{aligned}
P(\vec{p}) A(\vec{p})=(P A)(\vec{p}) & =\sum_{k=1}^{N} \mu_{p_{k}}(\vec{p}) P_{\vec{p}_{k}} A_{\vec{p}_{k}} \\
P(\vec{p}) B(\vec{p})=(P B)(\vec{p}) & =\sum_{k=1}^{N} \mu_{p_{k}}(\vec{p}) P_{\vec{p}_{k}} B_{\vec{p}_{k}} \\
C(\vec{p}) & =\sum_{k=1}^{N} \mu_{p_{k}}(\vec{p}) C_{\vec{p}_{k}} \\
D(\vec{p}) & =\sum_{k=1}^{N} \mu_{p_{k}}(\vec{p}) D_{\vec{p}_{k}} \\
P(\vec{p}) & =\sum_{k=1}^{N} \mu_{p_{k}}(\vec{p}) P_{\vec{p}_{k}}
\end{aligned}
$$

it is seen by inspection that the parameterization $(P A)(\vec{p}),(P B)(\vec{p}), C(\vec{p}), D(\vec{p}), P(\vec{p})$ thus obtained is passive. Since any non-negative linear combination of positive (negative) semi-definite real matrices is a positive (negative) semi-definite real matrix, stability and passivity are preserved over the entire design space. Similar results can be obtained for admittance and impedance representations [3].

The problem with the passive parameterized interpolation procedure for the Gilbert and balanced realizations are twofold. First, there are 5 interpolation equations (27) to be satisfied. Secondly the interpolation technique yields by construction the discrete macro-model $\mathscr{S}_{\vec{p}_{k}}$ for $\vec{p}=\vec{p}_{k}$, it is not at all sure that the interpolated matrices $A(\vec{p}), B(\vec{p}), C(\vec{p}), D(\vec{p})$ (or related descriptor form) will behave smoothly between the nodes $\vec{p}_{k}$. The reason for this is that minimal realizations are all equivalent modulo a similarity transformation, i.e., two realizations related by

$$
\left[\begin{array}{ll}
\tilde{A}_{\vec{p}_{k}} & \tilde{B}_{\vec{p}_{k}} \\
\tilde{C}_{\vec{p}_{k}} & \tilde{D}_{\vec{p}_{k}}
\end{array}\right]=\left[\begin{array}{cc}
X^{-1} A_{\vec{p}_{k}} X & X^{-1} B_{\vec{p}_{k}} \\
C_{\vec{p}_{k}} X & D_{\vec{p}_{k}}
\end{array}\right]
$$

where $X$ is any nonsingular matrix, yield the same transfer function

$H_{\vec{p}_{k}}(s)=C_{\vec{p}_{k}}\left(s I-A_{\vec{p}_{k}}\right)^{-1} B_{\vec{p}_{k}}+D_{\vec{p}_{k}}=\tilde{C}_{\vec{p}_{k}}\left(s I-\tilde{A}_{\vec{p}_{k}}\right)^{-1} \tilde{B}_{\vec{p}_{k}}+\tilde{D}_{\vec{p}_{k}}$

It is also important to note that the interpolation of statespace matrices allows a higher modeling capability than the interpolation of transfer functions [1], [2], but unfortunately these methods are sensitive to issues related to the smoothness of the state-space matrices as a function of the parameters.

In the next subsections, we describe the proposed Sylvester realization.

\section{Sylvester Realization}

For the Sylvester realization we propose the following statespace feedback realization with feedback matrix $F$ and pivot matrix $\tilde{A}$.

$$
\begin{aligned}
\dot{x} & =\tilde{A} x+\tilde{B}_{\vec{p}_{k}} v \\
y & =\hat{C}_{\vec{p}_{k}} x+\tilde{D}_{\vec{p}_{k}} v \\
v & =-F x+u
\end{aligned}
$$

where $\tilde{A}$ is a fixed $n \times n$ pivot matrix and $F$ is a fixed $m \times n$ state-space feedback matrix. This realization can be written as

$$
\mathscr{R}_{\vec{p}_{k}} \equiv\left[\begin{array}{cc}
\tilde{A}-\tilde{B}_{\vec{p}_{k}} F & \tilde{B}_{\vec{p}_{k}} \\
\hat{C}_{\vec{p}_{k}}-\tilde{D}_{\vec{p}_{k}} F & \tilde{D}_{\vec{p}_{k}}
\end{array}\right]=\left[\begin{array}{cc}
\tilde{A}-\tilde{B}_{\vec{p}_{k}} F & \tilde{B}_{\vec{p}_{k}} \\
\tilde{C}_{\vec{p}_{k}} & \tilde{D}_{\vec{p}_{k}}
\end{array}\right]
$$

For $\mathscr{R}_{\vec{p}_{k}}$ and $\mathscr{S}_{\vec{p}_{k}}$ to be equivalent requires the existence of nonsingular matrices $X_{k}$ such that

$$
\begin{aligned}
\tilde{A}-\tilde{B}_{\vec{p}_{k}} F & =X_{k}^{-1} A_{\vec{p}_{k}} X_{k} \\
\tilde{B}_{\vec{p}_{k}} & =X_{k}^{-1} B_{\vec{p}_{k}} \\
\tilde{C}_{\vec{p}_{k}} & =C_{\vec{p}_{k}} X_{k}
\end{aligned}
$$

is needed.

By eliminating (32b) from (32a) we obtain the Sylvester equation

$$
A_{\vec{p}_{k}} X_{k}-X_{k} \tilde{A}+B_{\vec{p}_{k}} F=0
$$

for the unknown matrix $X_{k}$. We need the following 
Theorem 1: The Sylvester equation (33) has a unique nonsingular solution $X_{k}$ provided the pair $\left(A_{\vec{p}_{k}}, B_{\vec{p}_{k}}\right)$ is controllable, the pair $(\tilde{A}, F)$ is observable, and the intersection of the eigenspectra of $A_{\vec{p}_{k}}$ and $\tilde{A}$ is empty.

Proof: See [24], [33].

Note that Sylvester equations are routinely solved by the Matlab function lyap and has a computational cost of $O\left(n^{3}\right)$.

Remark 1: The Sylvester realizations given the pivot matrix $\tilde{A}$ and feedback matrix $F$, are all unique by construction. For the choice of $\tilde{A}$ we can take a block-diagonal or blockJordan matrix [24], which never shares eigenvalues with any of the $A_{\vec{p}_{k}}$ matrices. This can be accomplished by choosing the eigenvalues of $\tilde{A}$ close to the imaginary axis (see also the numerical simulations), similarly to $A_{\vec{p}_{k}}^{\text {num }}$ and $A_{\vec{p}_{k}}^{\text {den }}$ in (11) and (12). The choice of $F$ is subject to the requirement that the pair $(\tilde{A}, F)$ has to be observable. In this paper we have considered $F$ as $B_{\vec{p}_{k}}^{n u m}=B_{\vec{p}_{k}}^{d e n}$ as described in Section III-C with proper transformation. More generally speaking, $F$ can be chosen quite freely, or its choice can be embedded in the overall Sylvester algorithm [34]. It is important to choose the $\tilde{A}$ and $F$ matrices properly to have a well-conditioned Sylvester equation solution.

The Sylvester realization state-space matrices generate their own LMIs as (20) for S-parameters and as in (21) for Yparameters, i.e;

$$
\left[\begin{array}{ccc}
\left(\tilde{A}-\tilde{B}_{\vec{p}_{k}} F\right)^{T} \tilde{P}_{\vec{p}_{k}}+\tilde{P}_{\vec{p}_{k}}\left(\tilde{A}-\tilde{B}_{\vec{p}_{k}} F\right) & \tilde{P}_{\vec{p}_{k}} \tilde{B}_{\vec{p}_{k}} & \tilde{C}_{\vec{p}_{k}}^{T} \\
\left(\tilde{P}_{\vec{p}_{k}} \tilde{B}_{\vec{p}_{k}}\right)^{T} & -I & \tilde{D}_{\vec{p}_{k}}^{T} \\
\tilde{C}_{\vec{p}_{k}} & \tilde{D}_{\vec{p}_{k}} & -I
\end{array}\right] \leq 0
$$

for S-parameters and

$$
\left[\begin{array}{cc}
\left(\tilde{A}-\tilde{B}_{\vec{p}_{k}} F\right)^{T} \tilde{P}_{\vec{p}_{k}}+\tilde{P}_{\vec{p}_{k}}\left(\tilde{A}-\tilde{B}_{\vec{p}_{k}} F\right) & \tilde{P}_{\vec{p}_{k}} \tilde{B}_{\vec{p}_{k}}-\tilde{C}_{\vec{p}_{k}}^{T} \\
\left(\tilde{P}_{\vec{p}_{k}} \tilde{B}_{\vec{p}_{k}}-\tilde{C}_{\vec{p}_{k}}^{T}\right)^{T} & -\tilde{D}_{\vec{p}_{k}}-\tilde{D}_{\vec{p}_{k}}^{T}
\end{array}\right] \leq 0
$$

for Y-parameters. The ARE can also be similarly be recast.

It is then parameterized as in the four last equations of (27), i.e.,

$$
\begin{aligned}
\tilde{P}(\vec{p}) \tilde{B}(\vec{p})=(\tilde{P B})(\vec{p}) & =\sum_{k=1}^{N} \mu_{k}(\vec{p}) \tilde{P}_{\vec{p}_{k}} \tilde{B}_{\vec{p}_{k}} \\
\tilde{C}(\vec{p}) & =\sum_{k=1}^{N} \mu_{k}(\vec{p}) \tilde{C}_{\vec{p}_{k}} \\
\tilde{D}(\vec{p}) & =\sum_{k=1}^{N} \mu_{k}(\vec{p}) \tilde{D}_{\vec{p}_{k}} \\
\tilde{P}(\vec{p}) & =\sum_{k=1}^{N} \mu_{k}(\vec{p}) \tilde{P}_{\vec{p}_{k}}
\end{aligned}
$$

The first equation of (27) has no counterpart in equations (35), since it is easy to show that

$$
(\tilde{P}[\tilde{A}-\tilde{B} F])(\vec{p})=\sum_{k=1}^{N} \mu_{p_{k}}(\vec{p}) \tilde{P}_{\vec{p}_{k}}\left[A-B\left(\vec{p}_{k}\right) F\right]
$$

Finally, the parameterized Sylvester realization in descriptor format is then simply generated by the interpolated matrices $(\tilde{P} \tilde{A})(\vec{p}),(\tilde{P} \tilde{B})(\vec{p}), \tilde{C}(\vec{p}), \tilde{D}(\vec{p}), \tilde{P}(\vec{p})$ and the parameterized transfer function is

$$
H_{\vec{p}}(s)=\tilde{C}_{\vec{p}}(s \tilde{P}(\vec{p})-\tilde{P}(\vec{p}) \tilde{A}(\vec{p}))^{-1} \tilde{P} \tilde{B}(\vec{p})+\tilde{D}(\vec{p})
$$

Remark 2: Note that, even if passivity is not required, the Sylvester realizations $\mathscr{R}_{\vec{p}_{k}}$ can be very useful for parameterization. Suppose the interpolation kernel $\mathscr{K}\left(\vec{p}_{k}, \vec{p}\right)=\mu_{p_{k}}(\vec{p})$ is not necessarily positive, but satisfies partition of unity i.e.,

$$
\sum_{p_{k}} \mu_{p_{k}}(\vec{p})=1, \quad \mu_{p_{k}}\left(\vec{p}_{l}\right)=\delta_{p_{k}, l}
$$

Then it is clear that the interpolation procedure

$$
\begin{aligned}
\tilde{B}(\vec{p}) & =\sum_{p_{k}=1}^{N} \mu_{p_{k}}(\vec{p}) \tilde{B}_{\vec{p}_{k}} \\
\tilde{C}(\vec{p}) & =\sum_{p_{k}=1}^{N} \mu_{p_{k}}(\vec{p}) \tilde{C}_{\vec{p}_{k}} \\
\tilde{D}(\vec{p}) & =\sum_{p_{k}=1}^{N} \mu_{p_{k}}(\vec{p}) \tilde{D}_{\vec{p}_{k}}
\end{aligned}
$$

is a very simple way to generate a parameterized macromodel.

\section{NUMERICAL EXAMPLES}

In the following examples, we show the importance of the state-space realization. We validate the novel Sylvester realization approach, by a comparison with the standard Gilbert, balanced and barycentric realizations. The pole placement approach described in Section III-C [24] is used for the barycentric realization as it gives more robust solution in comparison to the linear system approach [15].

\section{A. Two Coupled Microstrip with Variable Spacing (CM)}

Two coupled microstrips (length $\mathrm{L}=2 \mathrm{~cm}$ ) are considered in this example. The cross-section is shown in Fig.1. The

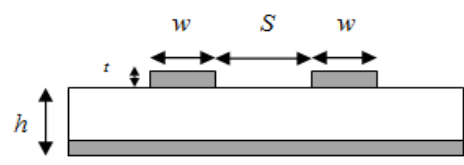

Fig. 1. CM: Two coupled microstrips.

conductors have a width $W$ of $500 \mu \mathrm{m}$ and the height $h$ of the dielectric is $800 \mu \mathrm{m}$. A bivariate macromodel is built as a function of the spacing $S$ between the microstrips and the frequency, freq. Their corresponding ranges are shown in Table I.

TABLE I

CM: PARAMETERS OF THE COUPLED MICROSTRIPS

\begin{tabular}{|l|l|l|}
\hline Parameter & Min & Max \\
\hline Frequency (freq) & $20 \mathrm{MHz}$ & $4 \mathrm{GHz}$ \\
Spacing $(S)$ & $1 \mathrm{~mm}$ & $3 \mathrm{~mm}$ \\
\hline
\end{tabular}

The scattering parameters were obtained by means of a fullwave solver based on the Partial Element Equivalent Circuit 
method [20] over a grid of $200 \times 15$ samples, for frequency and spacing respectively. We have built root macromodels for 8 values of the spacing by means of VF, each with an order equal to 8 .

As described in Section III-D, a pivot matrix and a feedback matrix must be chosen such that a well-conditioned solution is obtained for the Sylvester equation (33). We use a pivot matrix based on the set of poles $\left\{a_{n}\right\}_{n=1}^{N}$, chosen as follows

$$
a_{n}=-\alpha+j \beta, \quad a_{n}=-\alpha-j \beta
$$

where, $\alpha=\beta / 100$.

Also, since the eigenvalues of the pivot matrix and those of the root macromodels obtained from Gilbert realization must not be the same, we choose the poles very close to the imaginary axis as shown in Fig.2. The feedback matrix is chosen

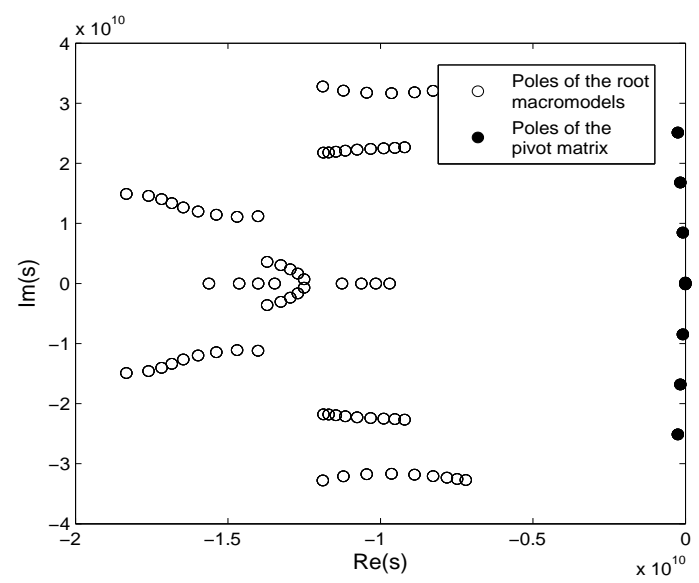

Fig. 2. CM: Eigenvalues of the pivot matrix and the root macromodels obtained from Gilbert realization.

as described in Section III-D. A similarity transformation is then performed using the Sylvester solution to obtain the statespace matrices of the Sylvester realization.

Then the four realizations are converted to a passive descriptor state-space form using LMI (34) as described in Section III with the help of CVX [35]. A bivariate macromodel is then obtained by linear interpolation of the corresponding statespace matrices. The parameterized macromodel obtained by the Sylvester realization is shown in Fig.3 .

Fig.4 compares $S_{11}(s, S)$ and its macromodel using the proposed realization for the spacing values $S=\{1.08,2.08,2.91\}$ $\mathrm{mm}$ that have not been used for the generation of the root macromodels.

The error plot in Fig.5 shows the absolute error at the validation points for the different realizations. It can be seen that the proposed Sylvester realization method gives a more accurate parametric macromodel with an absolute maximum error of $-55.77 \mathrm{~dB}$ in comparison with the other realization techniques. The error plot of barycentric realization and the proposed technique is almost similar, but the proposed

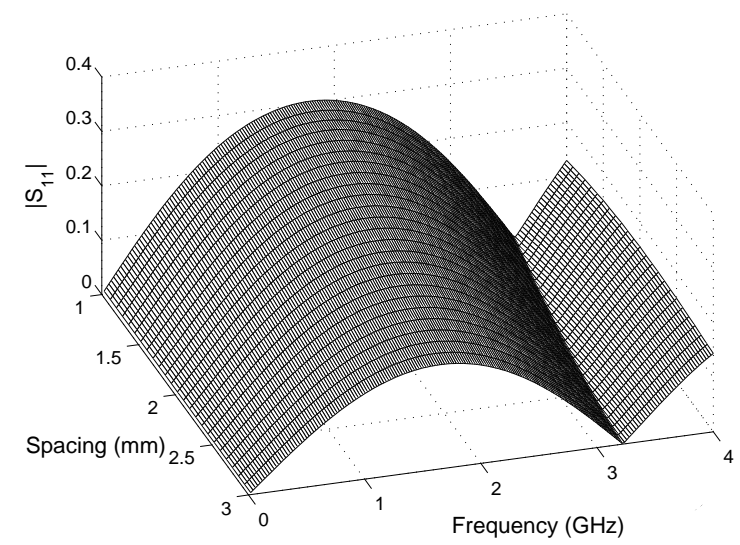

Fig. 3. CM: Magnitude of the bivariate macromodel $S_{11}(s, S)$ (Sylvester realization for each root macromodel).

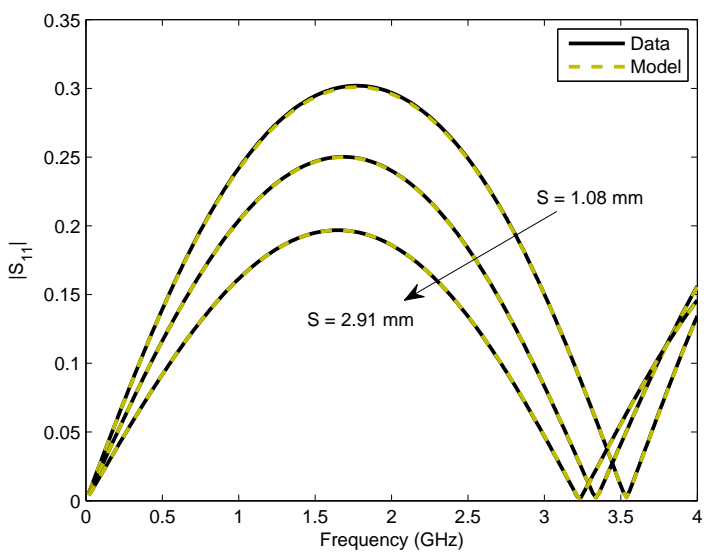

Fig. 4. CM: Magnitude of the bivariate macromodel of $S_{11}(s, S)(S=$ $\{1.08,2.08,2.91\} \mathrm{mm}$ using the Sylvester realization.

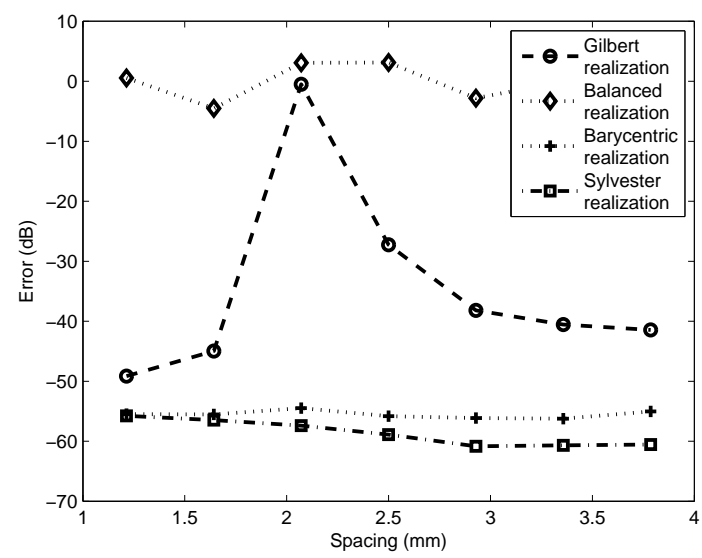

Fig. 5. CM: Error comparison for the different realizations.

technique is computationally cheaper in comparison with barycentric realization.

Table II compares the accuracy and CPU time needed for the four realizations. As the initial set of data samples are built using the VF technique, the root macromodels are in 
TABLE II

CM: COMPARISON OF THE DIFFERENT TECHNIQUES

\begin{tabular}{|l|c|c|}
\hline Technique & Maximum Error $(\mathrm{dB})$ & CPU time $(\mathrm{sec})$ \\
\hline Gilbert & -0.505 & - \\
Balanced & 3.134 & 0.115 \\
Barycentric & -55.032 & 0.281 \\
Sylvester & -55.766 & 0.095 \\
\hline
\end{tabular}

the standard Gilbert form. Thus II gives the CPU time for the transformation from the Gilbert state-space form to the respective state-space realization forms. It can be noted, that the proposed Sylvester realization is accurate and has the least computation.

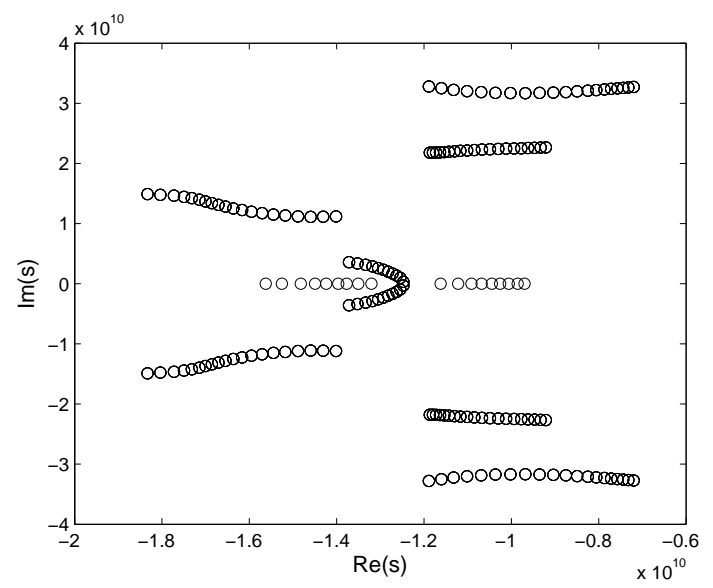

Fig. 6. CM: Model poles as a function of spacing for the root macromodels.

The distribution of the poles is shown in Fig.6; a bifurcation effect on a couple of poles is visible with two real poles moving towards each other and becoming a complex conjugate pole-pair, causing the slope of the corresponding trajectory to become discontinuous. This example shows that direct parameterization of the poles should be avoided due to potentially non-smooth behavior with respect to the design parameters. The effect of the bifurcation for the Gilbert realization can be seen in Fig.7.

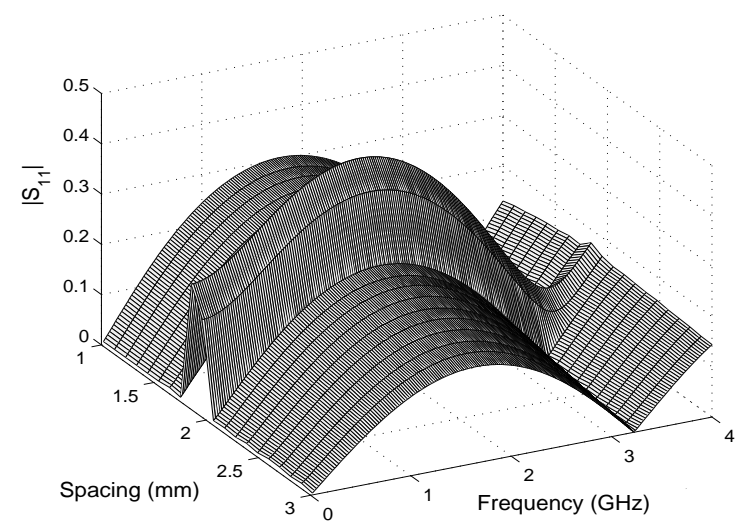

Fig. 7. CM: Magnitude of the bivariate macromodel $S_{11}(s, S)$ using the Gilbert realization.
In order to verify the properties of the parameterized macromodel using Sylvester realization, we check the poles of the parameterized macromodel over a dense sample (i.e; 200 samples) of the spacing $S$ and is seen in Fig.8 that the real part of the poles are negative and thus the system is stable.

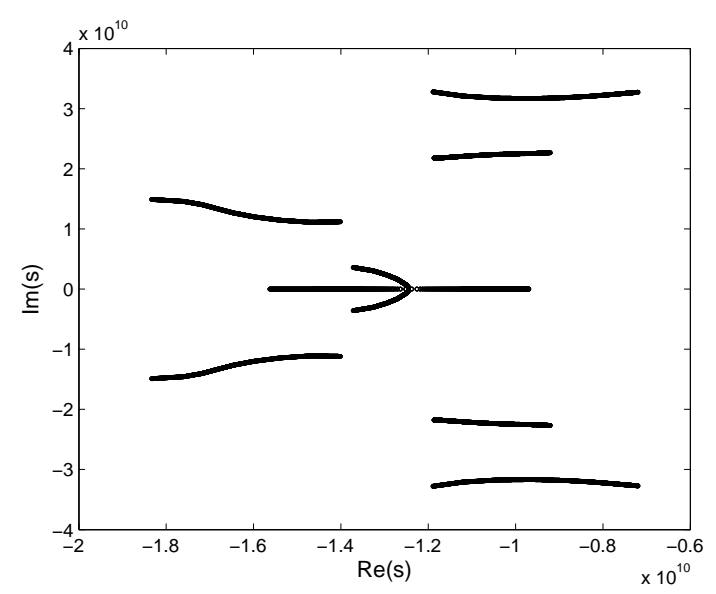

Fig. 8. CM: Poles of the bivariate macromodel $S(s, S)$ using the Sylvester realization.

The $H_{\infty}$ [36] of the system for the same dense samples of the spacing $S$ is plotted in Fig.9 and is found to be always less than 1 which shows that the system is passive.

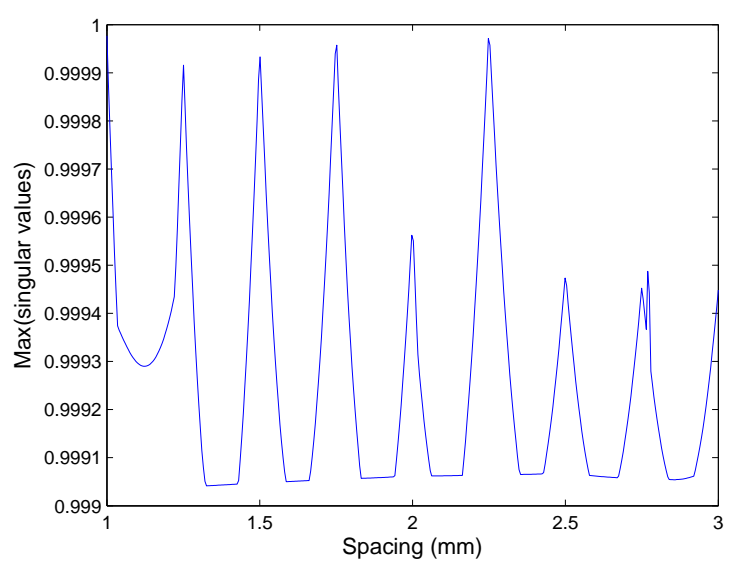

Fig. 9. CM: $H_{\infty}$ for the bivariate macromodel $S(s, S)$ uisng the Sylvester realization.

Thus the proposed Sylvester realization is able to provide a more accurate parameterized macromodel than the Gilbert realization and balanced realization, and a computationally cheaper model in comparison with barycentric realization. Also by using an LMI along with the Sylvester realization a stable and passive parameterized macromodel is obtained.

\section{B. Folded Stub Notch Filter with Variable Length and Variable Spacing (NF)}

A folded stub microwave notch filter on a substrate with relative permitivity $\varepsilon_{r}=9.6$ and a thickness of $0.635 \mathrm{~mm}$ is 
modeled in this example. The layout of this filter is shown in Fig.10.

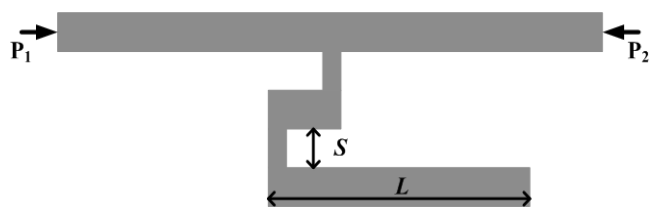

Fig. 10. NF: Layout of the folded stub notch filter.

The spacing $S$ and the length $L$ of the stub are chosen as design variables in addition to frequency. Their corresponding ranges are shown in Table III.

TABLE III

NF: PARAMETERS OF THE FOLDED STUB NOTCH FILTER

\begin{tabular}{|l|l|l|}
\hline Parameter & Min & Max \\
\hline Frequency $($ freq) & $2 \mathrm{GHz}$ & $4 \mathrm{GHz}$ \\
Length $(L)$ & $5 \mathrm{~mm}$ & $10 \mathrm{~mm}$ \\
Spacing $(S)$ & $0.5 \mathrm{~mm}$ & $0.7 \mathrm{~mm}$ \\
\hline
\end{tabular}

The scattering parameters were computed with advanced design system (ADS) momentum over a grid of $11 \times 11$ samples, for length and spacing respectively. We have built root macromodels for $6 \times 6$ values of the length and spacing respectively by means of VF, each with an order 6 .

As in the previous example, a pivot matrix and feedback matrix are found, and the eigenvalues of the pivot matrix and of the root macromodels are different as shown in Fig.11. Then a similarity transformation is done using the Sylvester

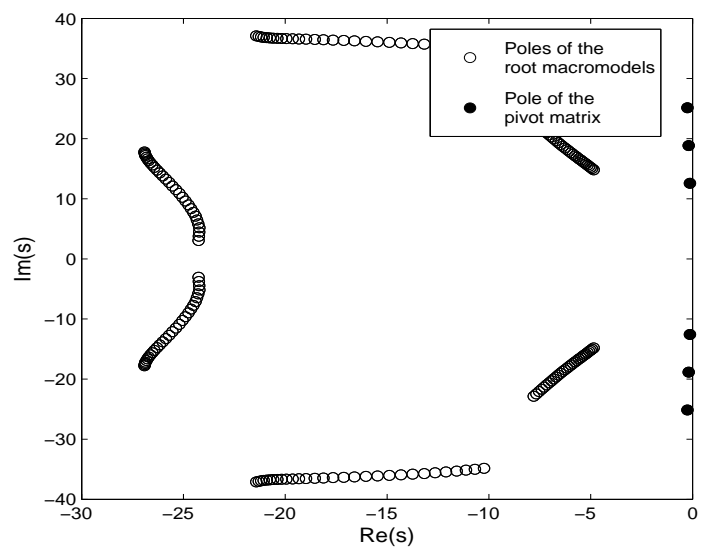

Fig. 11. NF: Eigenvalues of the pivot matrix and the root macromodels obtained from Gilbert realization.

solution to obtain the state-space matrices of the Sylvester realization. In order to convert the state-space matrices to a passive descriptor state-space form, we solve the LMI (34) using CVX [35] for the three realizations.

Thus, an accurate trivariate macromodel is obtained by the multilinear interpolation of the corresponding state-space matrices. Fig. 12 shows the parameterized macromodel obtained using the Sylvester realization .

Fig.13 compares $S_{12}(s, L, S)$ and its macromodel for the length values $L=\{5.5,6.5,7.5,8.5\} \mathrm{mm}$ that have not been

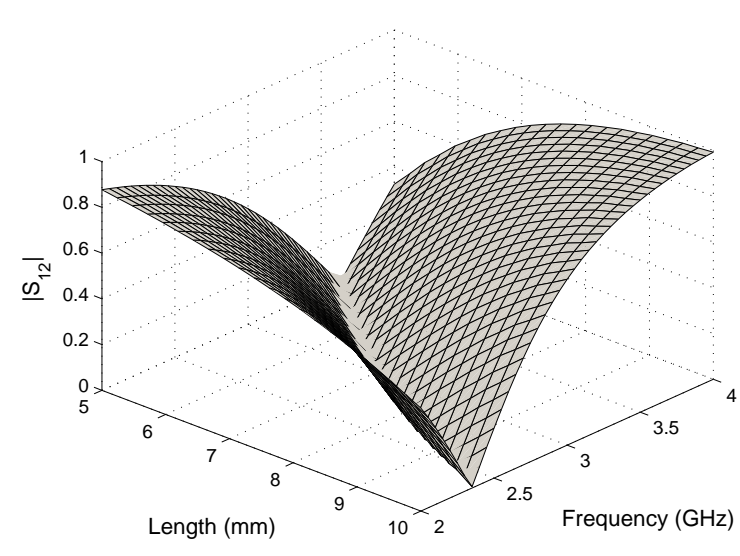

Fig. 12. NF: Magnitude of the trivariate macromodel $S_{12}(s, L, S)$ for $S=$ $0.5 \mathrm{~mm}$ using Sylvester realization.

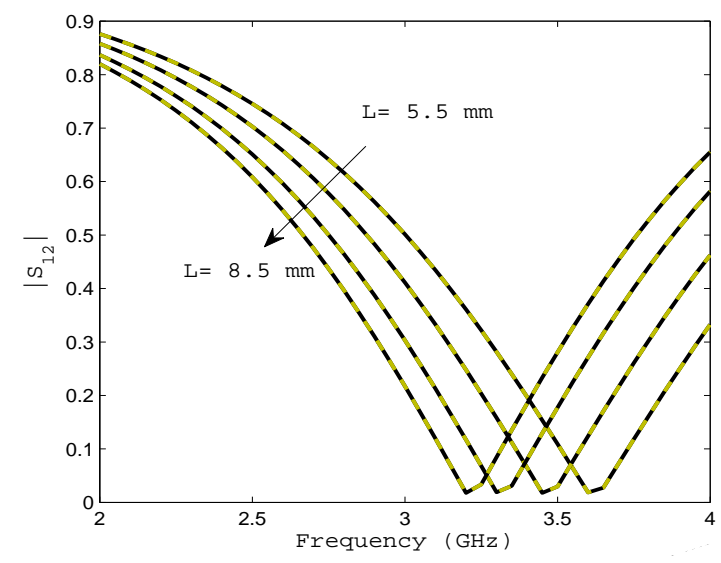

Fig. 13. NF: Magnitude of the trivariate macromodel for $S=0.6 \mathrm{~mm}$ of $S_{12}(s, L, S)(L=\{5.5,6.5,7.5,8.5\} \mathrm{mm})$ using Sylvester realization.

used for the generation of the root macromodels using the Sylvester realization.

As in the case of the two coupled microstrips, in this example also we can see from the error plot in Fig.14 that the proposed realization method gives an accurate parameterized macromodel with an absolute maximum error of $-43.62 \mathrm{~dB}$.

\section{TABLE IV}

NF: COMPARISON OF THE DIFFERENT TECHNIQUES

\begin{tabular}{|l|c|c|}
\hline Technique & Maximum Error (dB) & CPU time (sec) \\
\hline Gilbert & -21.201 & - \\
Balanced & 9.718 & 0.891 \\
Barycentric & -45.552 & 0.963 \\
Sylvester & -43.617 & 0.378 \\
\hline
\end{tabular}

While the barycentric realization provides a slightly better accuracy than the Sylvester-based realization, but it results to be computationally more expensive as shown in Table IV.

Fig. 15 shows the parameterized macromodel using balanced realization, and we note that the behavior is very erratic in comparison with Fig.12. This is due to the abrupt changes in the elements of the state-space matrices obtained through the balanced realization. 


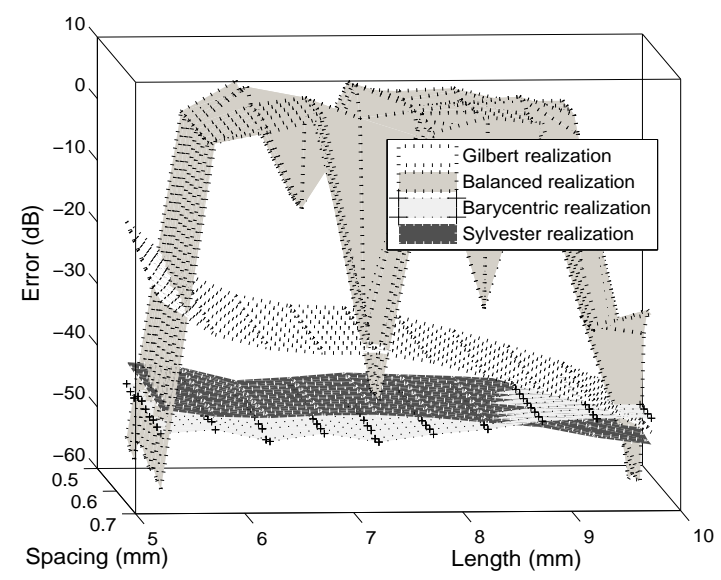

Fig. 14. NF: Error comparison for the different state-space realizations.

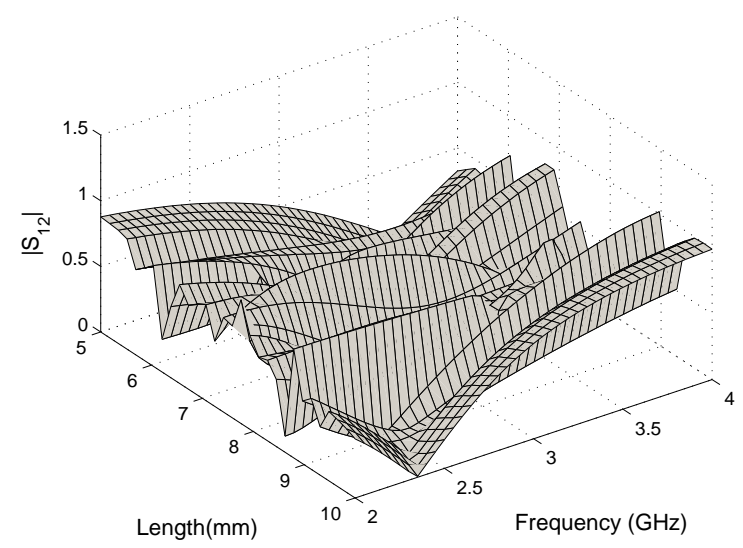

Fig. 15. NF: Magnitude of the trivariate macromodel $S_{12}(s, L, S)$ using balanced realization for each root macromodel).

The behavior of one of the elements in the matrix $A_{\overrightarrow{p_{k}}}$ is shown in Fig. 16. The figure compares the behavior of the corresponding element in Sylvester realization and can be seen to have a smooth variation.
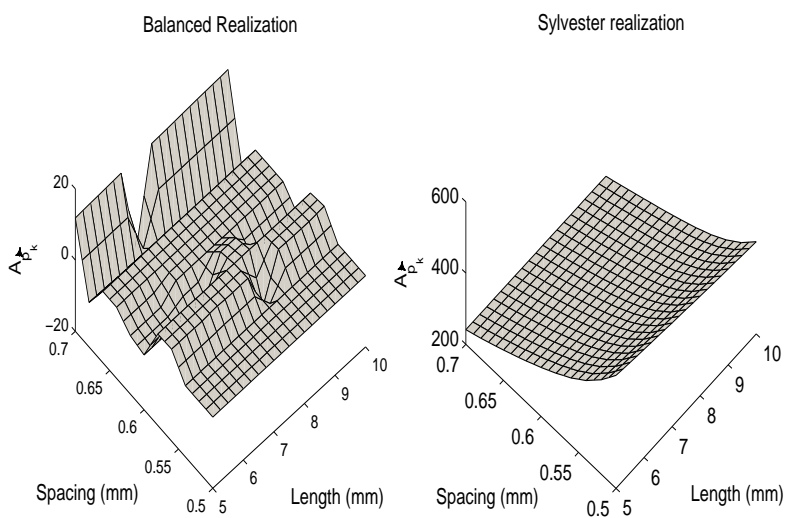

Fig. 16. NF: An entry of the $A_{\vec{p}}$.

In order to verify the stability and passivity of the parameterized macromodel using the Sylvester realization, we check its poles over a dense sampling (i.e; of $200 \times 200$ samples of the spacing $S$ and length $L$ ). In Fig.17 we can see that the real part of the poles is always negative and therefore the system is stable.

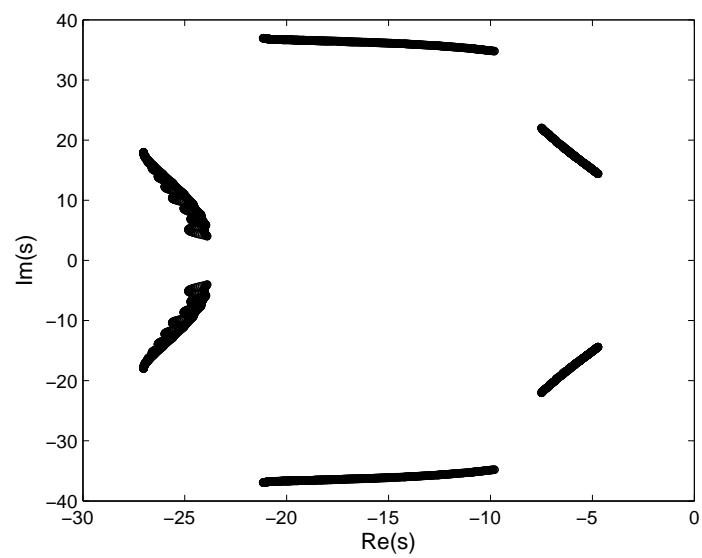

Fig. 17. NF: Poles of the trivariate macromodel $S(s, L, S)$ using Sylvester realization.

For passivity similar to the previous example, the $H_{\infty}$ of the system is plotted in Fig.18 and it is found to be less than 1 for all cases.

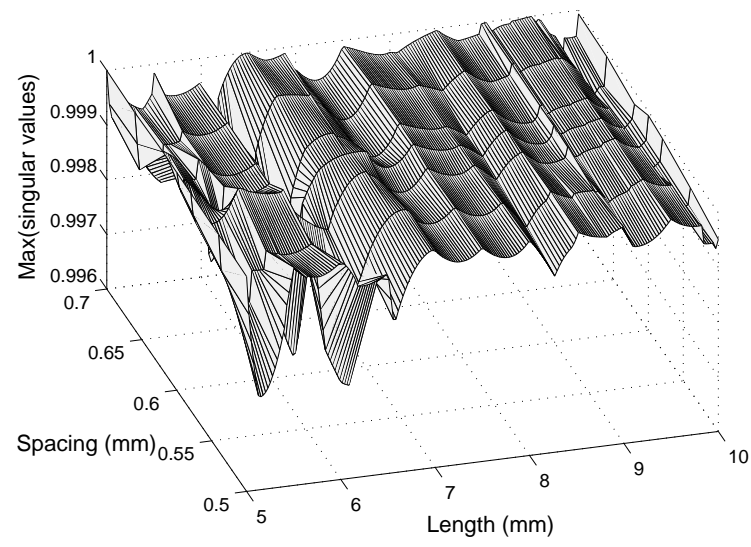

Fig. 18. NF: $H_{\infty}$ of the trivariate macromodel $S(s, L, S)$ using the Sylvester realization.

Thus the proposed Sylvester realization is able to provide a more accurate parameterized macromodel in comparison to Gilbert realization and balanced realization, and a computationally cheaper parameterized macromodel in comparison with barycentric realization. The stability and passivity of the system can be preserved by solving LMI and by using suitable interpolation schemes.

\section{CONClusion}

This paper proposes a novel state-space realization for parameterized macromodeling based on interpolation of statespace matrices. A judicious choice of the state-space realization is required to account for the generally assumed smoothness of the state-space matrices with respect to the parameters. 
Suitable interpolation schemes along with Sylvester realization are used to interpolate a set of root state-space matrices in order to build accurate parameterized macromodels. The essential aspects of this novel realization are to find a proper pivot matrix and to obtain a well-conditioned solution for a Sylvester equation. The numerical examples and related comparison results show that the proposed Sylvester realization provides very accurate parameterized macromodel with a low computation cost. The stability and passivity of the system can be preserved with the help of LMI and by the use of proper interpolation schemes.

\section{ACKNOWLEDGMENT}

This research has been funded by the Interuniversity Attraction Poles Programme BESTCOM initiated by the Belgian Science Policy Office.

\section{REFERENCES}

[1] F. Ferranti, L. Knockaert, and T. Dhaene, "Guaranteed passive parameterized admittance-based macromodeling," IEEE Transactions on Advanced Packaging, vol. 33, no. 3, pp. 623 -629, Aug. 2010.

[2] _ - "Parameterized S-parameter based macromodeling with guaranteed passivity," IEEE Microwave and Wireless Component Letters, vol. 19 , no. 10 , pp. $608-610$, Oct. 2009

[3] F. Ferranti, L. Knockaert, T. Dhaene, and G. Antonini, "Passivitypreserving parametric macromodeling for highly dynamic tabulated data based on Lur'e equations," IEEE Transactions on Microwave Theory and Techniques, vol. 58, no. 12, pp. 3688 -3696, Dec. 2010.

[4] P. Triverio, M. Nakhla, and S. Grivet-Talocia, "Passive parametric modeling of interconnects and packaging components from sampled impedance, admittance or scattering data," Electronic System-Integration Technology Conference, pp. 1-6, Sept. 2010.

[5] J. De Caigny, J. F. Camino, and J. Swevers, "Interpolating model identication for siso linear parameter-varying systems," Mechanical Systems and Signal Processing, vol. 23, no. 8, pp. 2395-2417, 2009.

[6] B. Gustavsen and A. Semlyen, "Rational approximation of frequency domain responses by vector fitting," IEEE Transaction on Power Delivery, vol. 14, pp. 1052-1061, Jul. 1999.

[7] T. Dhaene, D. Deschrijver, and N. Stevens, "Efficient algorithm for passivity enforcement of S-parameter bsed macromodels," IEEE Transactions on Microwave Theory and Techniques., vol. 57, no. 2, pp. 415420, Feb. 2009

[8] D. Saraswat, R. Achar, and M. Nakhla, "Global passivity enforcement algorithm for macromodels of interconnect subnetworks characterized by tabulated data," IEEE Transactions on Very Large Scale Integration (VLSI) Systems,, vol. 13, no. 7, pp. 819 -832, July 2005.

[9] S. Grivet-Talocia, "Passivity enforcement via perturbation of hamiltonian matrices," Circuits and Systems I: Regular Papers, IEEE Transactions on, vol. 51, no. 9, pp. 1755 - 1769, Sept. 2004.

[10] L. Knockaert, T. Dhaene, F. Ferranti, and D. D. Zutter, "Model order reduction with preservation of passivity, non-expansivity and markov moments." Systems \& Control Letters, vol. 60, no. 1, pp. 53-61, Jan. 2011.

[11] R. Pintelon, P. Guillaume, and Y. Rolain, "Parametric identification of transfer functions in the frequency domain- A survey," IEEE Transactions on Automatic Control, vol. 39, no. 11, pp. 2245-2260, Nov. 1994.

[12] K. Glover, "All optimal hankel-norm approximations of linear multivariable systems and their $\mathrm{L}^{\infty}$-error bounds," International Journal of Control, vol. 39, no. 6, pp. 1115-1193, 1984.

[13] Rolf Jeltsch and Mohamed Mansour, Stability Theory: Hurwitz Centenary Conference Centro Stefano Franscini, Ascona, 1995. Birkhäuser, 1996 edition (August 28, 1996).

[14] E. G. Gilbert, "Controllability and observability in multi-variable control systems," SIAM Journal on Control, vol. 1, no. 2, pp. 128-151, 1963.

[15] P. Triverio, S. Grivet-Talocia, and M. Nakhla, "A parameterized macromodeling strategy with uniform stability test," IEEE Transactions on Advanced Packaging, vol. 32, no. 1, pp. 205 -215, Feb. 2009.

[16] B. Moore, "Principal component analysis in linear systems: Controllability, observability, and model reduction," IEEE Transactions Automatic Control, vol. 26, no. 1, pp. 17-31, Feb. 1981.
[17] L. Pernebo and L. M. Silverman, "Model reduction via balanced state space representations." IEEE Transactions Automatic Control, vol. 27, no. 2 , pp. 382-387, 1982

[18] M. Lovera and G. Mercere, "Identification for gain-scheduling: a balanced subspace approach," American Control Conference, 2007., pp. 858 -863 , July 2007.

[19] R. Peeters, M. Olivi, and B. Hanzon, "Balanced realization of lossless systems: Schur parameters, canonical forms and applications," 15th IFAC Symposium on System Identification, pp. 273-283, 2009.

[20] F. Ferranti, L. Knockaert, T. Dhaene, and G. Antonini, "Parametric macromodeling for S-parameter data based on internal nonexpansivity," International Journal of Numerical Modelling: Electronic Networks, Devices and Fields, vol. 26, no. 1, pp. 15-27, 2013.

[21] L. Knockaert, F. Ferranti, and T. Dhaene, "Vector fitting vs. levenbergmarquardt : Some experiments," IEEE Workshop on Signal Propagation on Interconnects, 2009. SPI '09., pp. 1-4, May 2009.

[22] S. Skogestad and I. Postlethwaite, Multivariable Feedback Control Analysis and Design. Wiley, 1996.

[23] J. Kautsky, N. K. Nichols, and P. Van Dooren, "Robust pole assignment in linear state feedback," International Journal of Control, vol. 41, no. 5, pp. $1129-1155,1985$

[24] A. Varga, "Robust pole assignment via sylvester equation based state feedback parametrization," Proceedings of the IEEE International Symposium on Computer-Aided Control System Design, pp. 13-18, 2000.

[25] B. Anderson and S. Vongpanitlerd, Network Analysis and Synthesis. Englewood Cliffs, NJ.: Prentice-Hall, 1973.

[26] S. Boyd, L. El Ghaoui, E. Feron, and V. Balakrishnan, "Linear matrix inequalities in system and control theory," Philadelphia, PA:SIAM 1994

[27] P. Triverio, M. Nakhla, and S. Grivet-Talocia, "Passive parametric macromodeling from sampled frequency data," IEEE International Conference on Signal Propagation and Interconnects, pp. 117 -120, May 2010.

[28] P. Gahinet and P. Apkarian, "An LMI-based parametrization of all $H_{\infty}$ controllers with applications," Proceedings of the 32nd Conference on Decision and Control, pp. 656-661, Dec. 1993.

[29] P. Gahinet, A. Nemirovski, A. Laub, and M. Chilali, LMI Control Toolbox User's Guide. Version 1: The MathWorks, Inc., 1995.

[30] G. Balas, R. Chiang, A. Packard, and M. Safonov, Robust Control Toolbox User's Guide. Version 3: The MathWorks, Inc., 2005.

[31] F. Ferranti, L. Knockaert, and T. Dhaene, "Passivity-preserving parametric macromodeling by means of scaled and shifted state-space systems," IEEE Transactions on Microwave Theory and Techniques, vol. 59, no. 10 , pp. $2394-2403$, Oct. 2011

[32] G. Allasia, A class of interpolating positive linear operators: theoretical and computational aspects. In Recent Developments in Approximation Theory, Wavelets and Applications, Kluwer: : Dordrecht, Singh SP (ed.), 1995.

[33] E. de Souza and S. Bhattacharyya, "Controllability, observability and the solution of AX -XB = C," Linear Algebra and its Applications, vol. 39, pp. $167-188,1981$.

[34] J. Carvalho, K. Datta, and Y. Hong, "A new block algorithm for fullrank solution of the sylvester-observer equation," IEEE Transactions on Automatic Control, vol. 48, no. 12, pp. 2223 - 2228, Dec. 2003.

[35] M. Grant and S. Boyd, "CVX: Matlab software for disciplined convex programming (web page and software)," July 2008. [Online]. Available: http://www.stanford.edu/ boyd/cvx/

[36] R. A. Horn and C. R. Johnson, Matrix Analysis. Cambridge: Cambridge University Press, 1990.

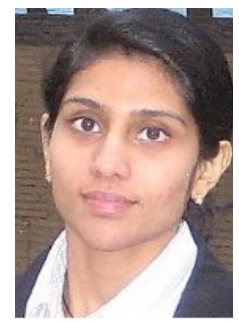

Elizabeth Rita Samuel received the Bachelor of Technology (B.Tech) degree and the Master of Technology (M.Tech) degree in Electrical and Electronics engineering from the University of Kerala, Kerala, India in 2002 and 2005 respectively. She is currently working toward the Ph.D. degree at the Department of Information Technology, Ghent University, Ghent, Belgium. Her current research interests include parametric macromodeling and parameterized model order reduction. 


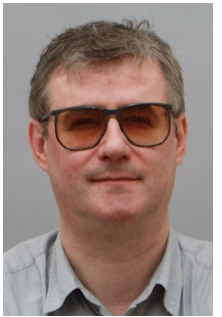

Luc Knockaert (SM'00) received the M. Sc. Degree in physical engineering, the M. Sc. Degree in telecommunications engineering and the $\mathrm{Ph}$. D. Degree in electrical engineering from Ghent University, Belgium, in 1974, 1977 and 1987, respectively. From 1979 to 1984 and from 1988 to 1995 he was working in North-South cooperation and development projects at the Universities of the Democratic Republic of the Congo and Burundi. He is presently affiliated with the Interdisciplinary Institute for Broad Band Technologies (www.ibbt.be) and a professor at the Dept. of Information Technology, Ghent University (www.intec.ugent.be). His current interests are the application of linear algebra and adaptive methods in signal estimation, model order reduction and computational electromagnetics. As author or co-author he has contributed to more than 100 international journal and conference publications. He is a member of MAA, SIAM and a senior member of IEEE.

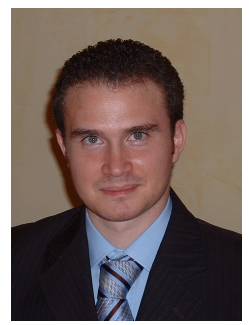

Francesco Ferranti (M'10) received the B.S. degree (summa cum laude) in electronic engineering from the Università degli Studi di Palermo, Palermo, Italy, in 2005, the M.S. degree (summa cum laude and honors) in electronic engineering from the Università degli Studi dell'Aquila, L'Aquila, Italy, in 2007, and the Ph.D. degree in electrical engineering from the University of Ghent, Ghent, Belgium, in 2011. He is currently a Post-Doctoral Research Fellow with the Department of Information Technology (INTEC), Ghent University, Ghent, Belgium. His research interests include parametric macromodeling, parameterized model order reduction, electromagnetic compatibility and signal integrity numerical modeling, stochastic modeling, system identification.

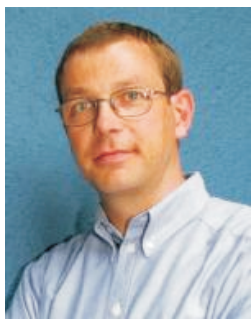

Tom Dhaene (SM'05) was born in Deinze, Belgium, on June 25, 1966. He received the Ph.D. degree in electrotechnical engineering from the University of Ghent, Ghent, Belgium, in 1993. From 1989 to 1993, he was Research Assistant at the University of Ghent, in the Department of Information Technology, where his research focused on different aspects of full-wave electro-magnetic circuit modeling, transient simulation, and time-domain characterization of high-frequency and high-speed interconnections. In 1993, he joined the EDA company Alphabit (now part of Agilent). He was one of the key developers of the planar EM simulator ADS Momentum. Since September 2000, he has been a Professor in the Department of Mathematics and Computer Science at the University of Antwerp, Antwerp, Belgium. Since October 2007, he is a Full Professor in the Department of Information Technology (INTEC) at Ghent University, Ghent, Belgium. As author or co-author, he has contributed to more than 250 peer-reviewed papers and abstracts in international conference proceedings, journals and books about computational science and engineering, numerical analysis, and computer science. He is the holder of 5 U.S. patents. 